Castro-Calviño, L., Rodríguez-Medina, J. \& López-Facal, R. (2021). Educación patrimonial para una ciudadanía participativa. Evaluación de resultados de aprendizaje del alumnado en el programa Patrimonializarte. Revista Electrónica Interuniversitaria de Formación del Profesorado, 24(1), 205-219.

\title{
Educación patrimonial para una ciudadanía participativa. Evaluación de resultados de aprendizaje del alumnado en el programa Patrimonializarte
}

\author{
Leticia Castro-Calviño', Jairo Rodríguez-Medina², Ramón López-Facal' \\ ${ }^{(1)}$ Departamento de Didácticas Aplicadas, Universidad de Santiago de Compostela \\ ${ }^{(2)}$ Departamento de Pedagogía, Universidad de Valladolid
}

\section{Resumen}

Se aborda la evaluación de programas de educación patrimonial, una materia pendiente en España. A partir de la implementación del programa Patrimonializarte y con el fin de valorar su eficacia, eficiencia y efectividad, se realiza la evaluación inicial, formativa y sumativa atendiendo a los resultados de aprendizaje de los 111 alumnos/as participantes. Se sigue un modelo de planificación y acción basado en el diseño y evaluación del programa que incorpora el trabajo sobre el patrimonio local y el uso de las TIC. Se analiza también la valoración del alumnado sobre la metodología utilizada. Se parte de la complementariedad metodológica basándose en el estudio múltiple de casos ( 6 grupos de 2 centros educativos en Galicia, España). Los principales resultados muestran: a) un importante impacto en la conciencia de conservación en el alumnado de educación infantil b) una creciente preocupación por el patrimonio intangible en educación primaria c) la importancia de la difusión para educación secundaria. El programa se considera pertinente, pues los alumnos/as han adquirido aprendizajes significativos para convertirse en ciudadanos que intervienen en el patrimonio de manera sostenible.

\section{Palabras clave}

Patrimonio local; patrimonio cultural; evaluación de programas; ciudadanía comprometida.

\section{Contacto:}

Castro-Calviño, L., leticiacastro.calvino@usc.es, Dpto. de Didácticas Aplicadas. Facultad de Ciencias de la Educación, Universidad de Santiago de Compostela, 15782 Santiago de Compostela., España.

Este artículo forma parte de los proyectos de investigación "Dimensiones emocionales y temas controvertidos en la formación del profesorado" (PGC2018-094491-B-C31) y "Conceptos metodológicos y métodos de aprendizaje activo para la mejora de las competencias docentes" (PGC2018-094491-B -C33), financiados por el Ministerio de Ciencia, Universidad e Innovación, cofinanciado por FEDER. 


\title{
Heritage education for participatory citizenship. Evaluation of student learning outcomes in the Patrimonializarte programme
}

\begin{abstract}
This paper examines the evaluation of heritage education programmes, a pending challenge in Spain. With the aim of evaluating the usefulness, efficiency and effectiveness of the Patrimonializarte programme, initial, formative and summative evaluations have been carried out with regard to the learning outcomes of the 111 pupils who participated in the programme. A planning and action model is followed based on the design and evaluation of the programme, which incorporates work on local heritage and the use of ICT resources. The evaluation made by the students of the methodology employed is also analysed. Methodological complementarity is assumed based on the study of multiple cases ( 6 groups from two schools in Galicia, Spain). The main results show: a) a significant impact on the awareness of conservation in early years pupils; b) a growing concern for intangible heritage in primary education; c) the importance of dissemination for secondary education. The programme is considered relevant due to the fact that the pupils have acquired significant learning with regard to becoming citizens who intervene in heritage in a sustainable way.
\end{abstract}

\section{Key words}

Local heritage; cultural heritage; programme evaluation; engaged citizenship.

\section{Introducción}

La investigación asume una concepción holística del patrimonio y la importancia de su estudio en el aula. Se plantea un modelo evaluativo para programas de educación patrimonial que contempla su multidimensionalidad (Pérez, 2006). Se diseña exprofeso el programa Patrimonializarte. Esta investigación parte de estudios que pretenden contribuir a este campo de estudio, todavía en fase de desarrollo en España (Castro-Calviño y López-Facal, 2019; Castro-Calviño, Rodríguez-Medina, Gómez-Carrasco, y López-Facal, 2020). En este artículo se presenta la evaluación inicial, formativa y sumativa del programa atendiendo a los resultados de aprendizaje del alumnado.

\section{Ciudadanía comprometida con su patrimonio}

El patrimonio es reconocido como un valor definitorio de identidad (Fontal, 2003). La identificación con el patrimonio y su difusión es importante para su reconocimiento y puesta en valor. Actualmente se aborda el patrimonio desde una perspectiva holística que aúna emociones e identidades sobre el entorno próximo para el desarrollo de una ciudadanía crítica, comprometida y concienciada con el desarrollo sostenible del patrimonio (CuencaLópez, Martín-Cáceres, y Estepa, 2020; Delgado-Algarra y Cuenca-López, 2020; MartínCáceres y Cuenca-López, 2015).

En la Delhi Declaration on Heritage and Democracy se incide en la importancia de la participación ciudadana para la gestión sostenible del patrimonio (ICOMOS, 2017). La colectividad y el valor concedido a lo local, pasa por una educación basada en los lugares. Este enfoque inspira la estrategia Place-Based Education (PBE) que pretende educar para la sostenibilidad poniendo en valor el espacio comunitario en el que se encuentre la escuela (Sánchez y Murga-Menoyo, 2019; Woodhouse y Knapp, 2000). El sentido de pertenencia a un lugar y de valoración de lo propio crea vínculos con los elementos patrimoniales (Pinto y 
Zarbato, 2017). El patrimonio juega un papel activo en las comunidades que se involucran y participan en su preservación y gestión (Marqués, Castro-Fernández, y López-Facal, 2020).

Un estudio reciente en España e Inglaterra, con futuros profesores de historia, muestra que no conceden demasiado valor a la historia local y al patrimonio intangible (Gómez-Carrasco, Miralles-Martínez, Fontal, e Ibáñez-Etxeberria, 2020). Se considera relevante trabajar en esta línea, pues la inclusión del patrimonio inmaterial en los programas educativos es fundamental para su salvaguarda (UNESCO, 2003; Kirshenblatt-Gimblett, 2004; Lenzerini, 2011; Yang, Shafi, Song y Yang, 2018).

El estudio del patrimonio desde edades tempranas puede contribuir a favorecer aprendizajes sociales significativos. La educación infantil (en adelante El) ofrece la oportunidad de comenzar a percibir una identidad compartida que favorece la valoración y conciencia de conservación sobre bienes patrimoniales próximos (Peinado, 2020). Salir del aula para conocer el patrimonio próximo ayuda a desarrollar habilidades y conocimientos desde un aprendizaje vivencial (Monti et al., 2017). La conciencia de compartir un patrimonio con los demás se amplía en la educación primaria (en adelante EP) desencadenando procesos de patrimonialización e identificación con el patrimonio próximo (Domínguez y López-Facal, 2015; Fontal, Ibáñez-Etxeberria, Martínez, y Rivero, 2017). El uso del patrimonio como recurso didáctico está más extendido en la educación secundaria (en adelante ES) en la que las metodologías activas ayudan a formar una ciudadanía crítica y responsable con el patrimonio (Fontal, 2016; Trabajo-Rite y Cuenca-López, 2020). Se considera importante que la vinculación con el patrimonio parta de la enseñanza formal y desde edades tempranas.

\section{Evaluación de programas de educación patrimonial}

Estudios realizados en España entre el profesorado en formación y en activo sobre la enseñanza del patrimonio muestran que sus concepciones se limitan a una dimensión histórico-artística (Cuenca-López, 2003; Jiménez-Pérez, Cuenca-López, y Ferreras-Listán, 2010). Esto también es habitual en los currículos educativos y en los libros de texto (CuencaLópez, Estepa-Giménez, y Martín-Cáceres, 2017; Cuenca-López y López-Cruz, 2014; Estepa, Ferreras, López, y Morón, 2011).

Las investigaciones sobre programas de educación patrimonial constatan deficiencias en los contenidos y en la evaluación (Fontal y García, 2019; Fontal, García, Arias, y Arias, 2019; Fontal y Martínez, 2017; Fontal, García, Arias y Arias, 2019). Para tratar de superar estas limitaciones se necesitan modelos de evaluación de programas. Se ha avanzado en la evaluación de programas en museos (Calaf, Gutiérrez, y Suárez, 2020) pero en la educación formal todavía se encuentra en un estadio inicial (Fontal, 2016; Sánchez-Macías, Fontal, y Rodríguez-Medina, 2019). Para contribuir a su desarrollo se diseña un programa basado en el estudio del patrimonio local que, además, incorpora metodologías activas de trabajo con TIC. EI programa internivel Patrimonializarte es integrado, integral e integrador y su diseño y evaluación se apoya en líneas de investigación emergentes (Pérez, 2006; Escudero, 2016).

\section{Objetivos de la investigación}

El objetivo principal es conocer los resultados de aprendizaje del alumnado en el programa Patrimonializarte para valorar la eficacia, eficiencia y efectividad y tomar decisiones orientadas a la mejora educativa (Pérez, 2002). Para responder a ello se plantean los siguientes objetivos específicos:

OE1. Comprobar si el alumnado ha adquirido aprendizajes significativos.

OE2. Acreditar las valoraciones del alumnado sobre las metodologías utilizadas. 


\section{Metodología}

Se trata de una investigación evaluativa de un estudio colectivo de casos en el se opta por el principio de complementariedad metodológica combinando métodos cuantitativos y cualitativos (Cook y Reichardt, 1982; Stake, 1995). Se planifica el programa en dos fases: en la primera se aplica la evaluación inicial y formativa, en la segunda la evaluación sumativa. El alumnado, como agente interesado en el proceso, valora también la metodología utilizada (Fetterman, Kaftarian, y Wandersman, 2015).

\section{Contexto y participantes}

El programa se implementa en los niveles educativos de EI, EP y ES de dos centros de la provincia de A Coruña (Galicia, España). El primero (Centro 1) es un Colegio Público Integrado $(\mathrm{CPI})$ en el que se imparten los tres niveles educativos. El segundo (Centro 2 ) es un Colegio de Educación Infantil y Primaria (CEIP). Entre los dos participan 111 escolares: 39 de El $\left(6^{\circ}\right.$ curso) 60 de EP ( 25 en $2^{\circ}$ Curso, 25 en $3^{\text {a }}$ y 10 en $5^{\circ}$ ) y 12 de ES ( $2^{\circ}$ curso). En la primera fase participan los 111, en la segunda lo hacen 86 pues no participa la clase de 25 alumnos/as de $3^{\circ}$ de primaria.

\section{Actividades}

En la Fase 1 se llevan a cabo actividades relacionadas con el patrimonio inmaterial (formas de vida y entornos pasados a través de la fotografía). En la segunda fase se trabaja con el patrimonio local olvidado y el patrimonio intangible: música, toponimia y patrimonio arqueológico. Las actividades se adecúan a cada nivel educativo. En El se combinan juegos y expresión artística para favorecer la motivación y la asimilación significativa de contenidos. En EP se fomenta la motivación hacia el patrimonio con salidas de campo. Se potencia el protagonismo del alumnado con recursos digitales que les permiten realizar creaciones audiovisuales propias. En ES se utiliza una metodología basada en el trabajo con softwares de geolocalización que les permiten crear contenidos digitales.

\section{Instrumentos y análisis de datos}

Se diseñan instrumentos diferenciados a las distintas edades del alumnado 1 . Para el análisis de datos se utilizan los programas SPSS v.26, R 3.6.3 y Atlas.ti v.8.4.4 (1135).

Los datos cuantitativos se tratan con una metodología analítica descriptiva de frecuencias y distribución porcentual. Se realizaron pruebas de McNemar para comprobar si existen diferencias significativas entre pre-test y post-test en cada una de las respuestas. Los datos cualitativos se analizan a partir del enraizamiento de códigos ( $\mathrm{Gr}=$ =número de citas vinculadas a un código). Esto se lleva a cabo también en el análisis proyectivo gráfico de El y en las videocápsulas de ES. Se establece un sistema de categorización para facilitar el análisis de datos (Tabla 1).

La complementación de diferentes técnicas analíticas permite ofrecer una información completa y rica en matices para conocer en profundidad el programa implementado (Greene, 2005; Johnson y Onwuegbuzie, 2004).

\footnotetext{
1 Las actividades, instrumentos y sistemas de categorización pueden consultarse bajo petición en la siguiente página web: http://www.rodausc.gal/novas/educacion-patrimonial-para-una-ciudadania-participativa-evaluacionde-resultados-de-aprendizaje-del-alumnado-en-el-programa-patrimonializarte/
} 
Tabla 1.

Categorías de análisis (no se incluyen las variables porque son comunes: V1:Nivel alto; V2: Nivel intermedio; V3: Nivel bajo).

\begin{tabular}{l|l}
\hline \multicolumn{1}{c|}{ Educación infantil } & Educación primaria y secundaria \\
\hline Entrevistas & Cuestionarios \\
\hline $\begin{array}{l}\text { C1: Exploración del entorno y formas de } \\
\text { vida en el pasado y el presente }\end{array}$ & C1: Percepciones sobre el patrimonio \\
\hline $\begin{array}{l}\text { C2: Paso del tiempo: cambios y } \\
\text { continuidades }\end{array}$ & C2: Cambios y continuidades \\
\hline C3: Capacidades afectivas y emociones & C3: Valoración del patrimonio \\
\hline C4: Valoración de la metodología utilizada & C4: Aprendizajes significativos \\
\hline \multicolumn{2}{c}{ Fación sumativa } \\
\hline Entrevista & Cuestionarios \\
\hline C1: Conocimientos adquiridos & C1: Conocimientos adquiridos \\
\hline $\begin{array}{l}\text { C2: Paso del tiempo: cambios y } \\
\text { continuidades }\end{array}$ & C2: Conciencia de conservación \\
\hline C3: Capacidades afectivas y emociones & C3: Capacidades afectivas y emociones \\
\hline C4: Valoración de la metodología utilizada & C4: Valoración de la metodología utilizada \\
\hline Test proyectivo gráfico & Texto reflexivo (EP) y video-cápsula (ES) \\
\hline $\begin{array}{l}\text { C1: Percepciones sobre el estado de } \\
\text { conservación del patrimonio }\end{array}$ & $\begin{array}{l}\text { C1: Conocimientos adquiridos sobre el } \\
\text { patrimonio próximo }\end{array}$ \\
\hline $\begin{array}{l}\text { C2: Expectativas sobre la conservación del } \\
\text { patrimonio }\end{array}$ & C2: Conciencia de conservación \\
\hline & C3: Capacidades afectivas y emociones \\
\hline
\end{tabular}

\section{Resultados}

\section{Educación Infantil}

- Evaluación inicial y formativa

En la Fase 1, se analizan los datos extraídos de las entrevistas grupales pre-test (evaluación inicial) y post-test (evaluación formativa) (Figura 1).

En el pre-test no encontramos registros de niveles altos en las categorías $\mathrm{C}_{1}$ y $\mathrm{C}_{2}$, y muy pocos en la C3. En la categoría C1 muestran un nivel de conocimientos intermedio ( 1 1.V2.PRE: $\mathrm{Gr}=30$ ) y bajo (C1.V3.PRE: $G r=39)$. 


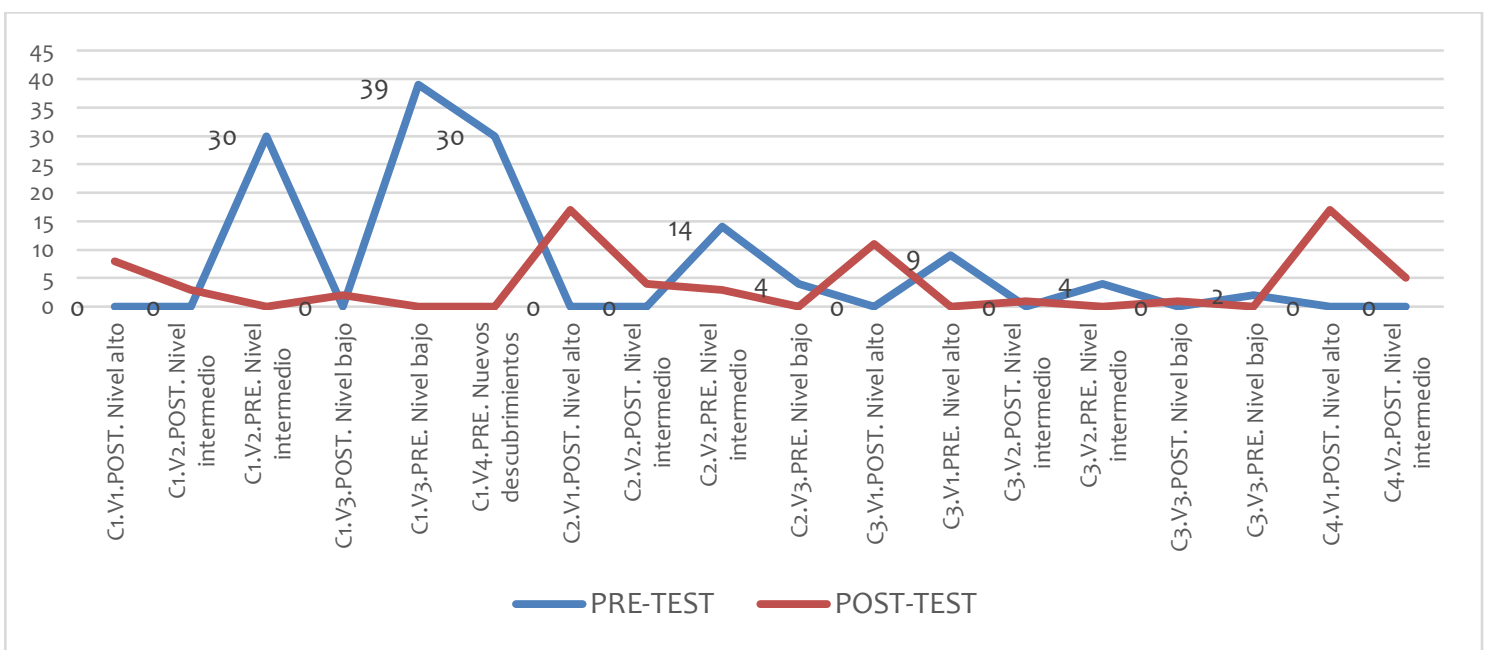

Figura 1. Enraizamiento de códigos (Gr) del pre-test y el post-test en educación infantil

En la sesión inicial ya adquieren nuevos conocimientos (conocen algunas de las principales diferencias entre las escuelas del pasado y las de ahora) (C1.V4.PRE: $\mathrm{Gr}=30$ ).

En la categoría C2 se constata un nivel intermedio (C2.V2.PRE: $\mathrm{Gr}=14$ ) y bajo (C2.V3.PRE: $\mathrm{Gr}=4)$. El alumnado empieza a reconocer el paso del tiempo y los cambios y continuidades entre su escuela con la de sus abuelos.

En la primera sesión se observa ya un nivel alto (C3.V1.PRE: Gr=9) e intermedio (C3.V2.PRE: $\mathrm{Gr}=4$ ) en la C3. Manifiestan que prefieren su escuela a la de sus abuelos/as, justificándolo "porque estamos juntos" (Cita 2:87) o "porque no tenemos que trabajar sin parar" (Cita: $2: 88)$. Tras analizar las fotografías, prefieren la escuela actual, aunque hay excepciones (EF.C3.V3.PRE: Gr:2).

En el post-test se alcanza un nivel alto de conocimientos adquiridos (C1.V1.POST: $\mathrm{Gr}=8$ ) identifican las principales diferencias entre la escuela del pasado y la actual. Se adquiere un nivel alto en la categoría C2 (C2.V1.POST: $G r=17)$. Desarrollan capacidades afectivas y emocionales ( $C_{3}$.V1.POST: $G r=11$ ) que expresan prefiriendo su escuela actual, porque reconocen que sus abuelos no tenían las comodidades que ellos tienen o que la segregación por sexos "da pena porque las chicas y chicos no pueden estar juntos"(Cita 7:8). Muestran una valoración alta en la C4 (C4.V1.POST: Gr=17) e intermedia (C4.V2.POST: Gr=5) debido a la vergüenza que pasaron hablando en público.

- Evaluación sumativa

Los resultados de la entrevista grupal de la Fase 2 se muestran en la Figura 2. No se encuentran niveles bajos en la categoría C2 ni intermedios en la categoría C4.
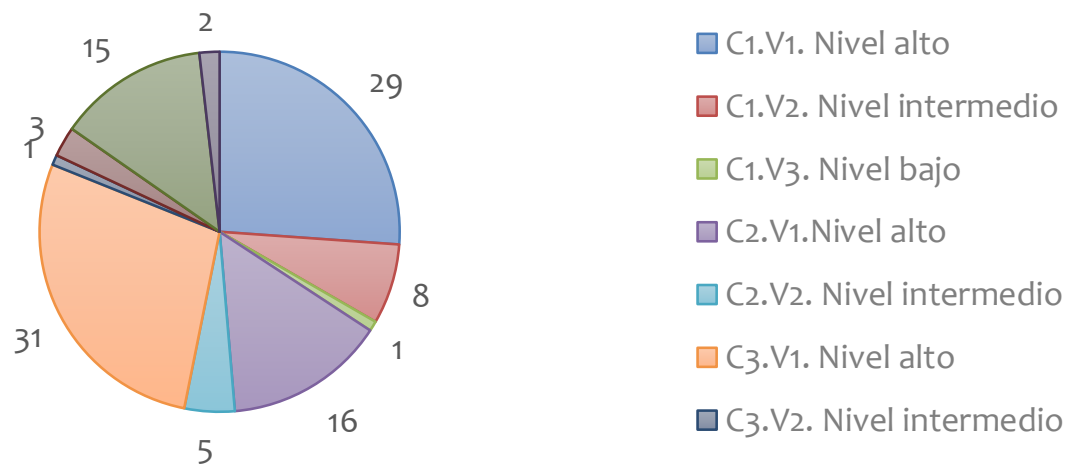

Figura 2. Enraizamiento de códigos (Gr) de la entrevista grupal de educación infantil 
En la categoría C1 se recoge un nivel alto (C1.V1: $\mathrm{Gr}=29)$ e intermedio ( $\mathrm{C}$.V2: $\mathrm{Gr}=8)$ Reconocen la función del dolmen y manifiestan que es "para cuidarlo" (Cita 49:5) y "no para subirse en el" (Cita 49:4). Se constata un nivel de conciencia de conservación alto ( $C_{2} . V_{1}:$ Gr=16). Mencionan el estado de conservación del dolmen: "está mal cuidado" (cita 48:3); "lo escavaron para buscar tesoros" (cita 49:42); "antes había un pasillo" (cita 49:54). Las capacidades afectivas y emociones alcanzan un nivel alto (C3.v1: Gr=31). La valoración de la metodología utilizada es alta ( $\left.\mathrm{C}_{4} \cdot \mathrm{V} 1: \mathrm{Gr}=15\right)$. Cuando se pregunta si les gustaría trabajar más sobre el patrimonio la respuesta es unánimemente afirmativa.

Por último, se analizan los datos extraídos del test proyectivo gráfico (Figura 5). Se responde con dibujos a las preguntas: ¿Qué hay en el dolmen que está mal? (C1) y ¿Cómo estaría el dolmen mejor? (C2).

En la categoría C1 destacan las alusiones a la conservación ( $\mathrm{C}$ 1.V1: $\mathrm{Gr}=25)$ y a la acción humana (C1.V2: Gr=11). Dibujan las piedras del dolmen tiradas; maleza; piedras rotas o gente subiéndose al bien patrimonial (Figuras 3 y 4). Se encuentra también información adicional ( $\mathrm{C}_{1} \mathrm{~V}_{3}$ : $\left.\mathrm{Gr}=2\right)$ : dos alumnos dibujan una grieta en la piedra de cubierta muy parecida a la que se encuentra en el dolmen.
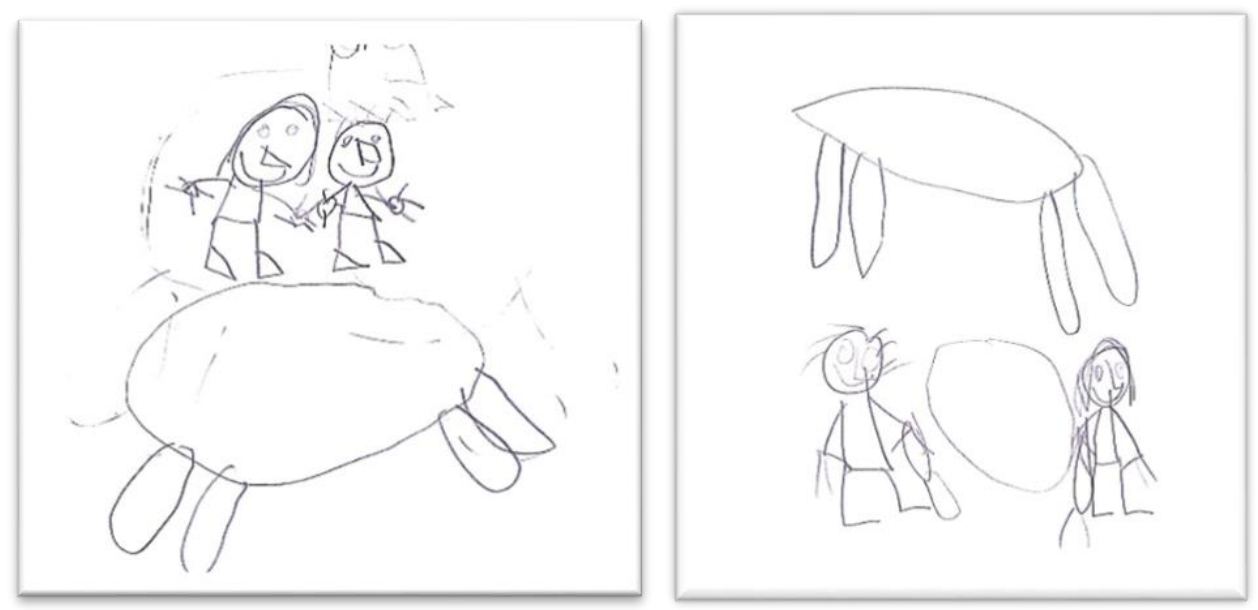

Figuras 3 y 4. C1: Perspectivas sobre la conservación del patrimonio. Dibujo A: gente subida encima del dolmen; Dibujo B: gente escavando y robando piedras

En la categoría C2, vuelve a destacar la conservación (C2.V1: Gr=36). Dibujan el dolmen con todas sus piedras, el entorno bien cuidado o carteles indicativos. Algunos alumnos dibujan información adicional que han adquirido en el desarrollo de las actividades como un corredor que ya no se conserva y daba acceso al dolmen originalmente (C2.V3: Gr=8) (Figura 5).

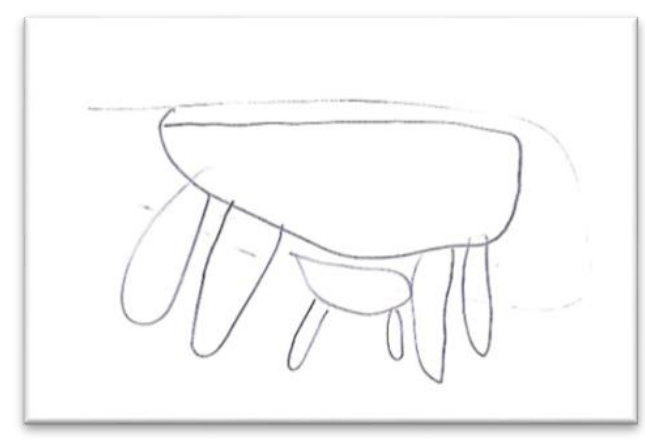

Figura 5. C2: Expectativas sobre la conservación del patrimonio. Dibujo del corredor original del dolmen. 


\section{Educación Primaria}

En la fase 1, los resultados de EP (60 alumnos/as) pueden ser comparados entre sí e incluso agregados porque coinciden entre pre-test y post-test (categorías 1-3).

- Evaluación inicial y formativa

En la categoría C1 ha disminuido el número de participantes que no responden (del 13,2\% al $0 \%$; se incrementa el de los que valoran que lo más importante son los monumentos y edificios porque atraen visitantes (pasan del $2 \%$ a más del $25 \% ; \chi^{2}(1)=10.28, p=.0013$ ) y se reduce el de los que valoran los aspectos paisajísticos del entorno (del 30,2\% al 13,8\%; $\chi_{2}(1)=$ $6.23, p=.012)$.

En la C2 aumenta el conocimiento sobre la desaparición de antiguos edificios del pueblo (pre $35.3 \%$, post $70.2 \% ; \chi_{2}(1)=11.56, p<.001$ ) y se reduce el número de alumnos/as que piensan que el único cambio fue en el edificio de la escuela, pero no la manera de impartir las clases (pre 19.6\%, post $5.3 \% ; \chi_{2}(1)=8, p=.004$ ). También disminuye el número de quienes piensan que no hubo cambios destacables (pre $7.8 \%$, post $0 \%$ ).

En la pregunta: ¿qué debe hacerse con los edificios antiguos?, se muestra una reducción no significativa del porcentaje entre pre-test y post-test de alumnos/as que prefieren conservarlos y darles nuevo uso (del 30,2\% al 28,1\%; $\chi_{2}(1)=.2, p=.654$ ) así como los que piensan que es mejor construir edificios nuevos (del $5,7 \%$ al $5,3 \% ; \chi_{2}(1)=.22, p=.637$ ). Sube el número de los que consideran preferible conservarlos y conocerlos para saber cómo vivían sus antepasados (del 64,2\% al 66,7\%; $\chi 2(1)=.6, p=.438$ ).

No se aprecian diferencias significativas entre cursos sobre cómo valoran las fotografías de cuando ellos eran más pequeños $\left(C_{3}\right)\left(\chi_{2}(4)=4 \cdot 9, p=.297\right)$. En cambio, sí existen diferencias respecto a la importancia y sentido que otorgan a las fotografías antiguas. Se incrementa el número de los que creen que cuentan cómo era la vida el pueblo en el pasado (pre $=8.08 \%$, post $\left.=17.17 \%, \chi_{2}(1)=5.4, p=.02\right)$. Disminuye el porcentaje de quienes las consideran sólo son recuerdos ( $p r e=79.2 \%$, post $=65.5 \%, \chi 2(1)=2.88, p=.089$ ) y también de los que no saben que en su casa se conservan fotografías antiguas ( $p r e=22 \%$, post $=12.1 \%, \chi_{2}(1)=3, p=.083$ ).

La categoría $C_{5}$ sólo se ha incluido en el post-test. Casi la mitad opta por respuestas vinculadas al contenido de la actividad (el $46 \%$ manifiesta que conoció cambios en la escuela y en los edificios) y la otra mitad considera que aprendieron a reconocer el potencial de la propia metodología (el $52 \%$ se dio cuenta de que las fotografías ayudan a conocer nuestro pasado). La salida de campo para realizar fotografías ha sido la actividad por la que han mostrado mayor satisfacción.

Existe unanimidad en valorar positivamente la actividad de presentación del trabajo realizado a otros grupos. El 53\% opina que los grupos que vinieron a enseñarles su trabajo realizaron un muy buen trabajo. Tres cuartas partes dicen que cuando se encargaron ellos explicar su trabajo, enseñaron cosas que los otros grupos no conocían. Casi dos terceras partes del alumnado enseñaron a su familia en la exposición su trabajo (15\%) y el del grupo (45\%). La satisfacción con la actividad de la exposición fue elevada, aunque el 37,9\% de las familias no pudieron asistir.

- Evaluación sumativa

En la Fase 2 se evalúan, con un cuestionario de preguntas abiertas, los conocimientos adquiridos durante el programa (Figura 6). 


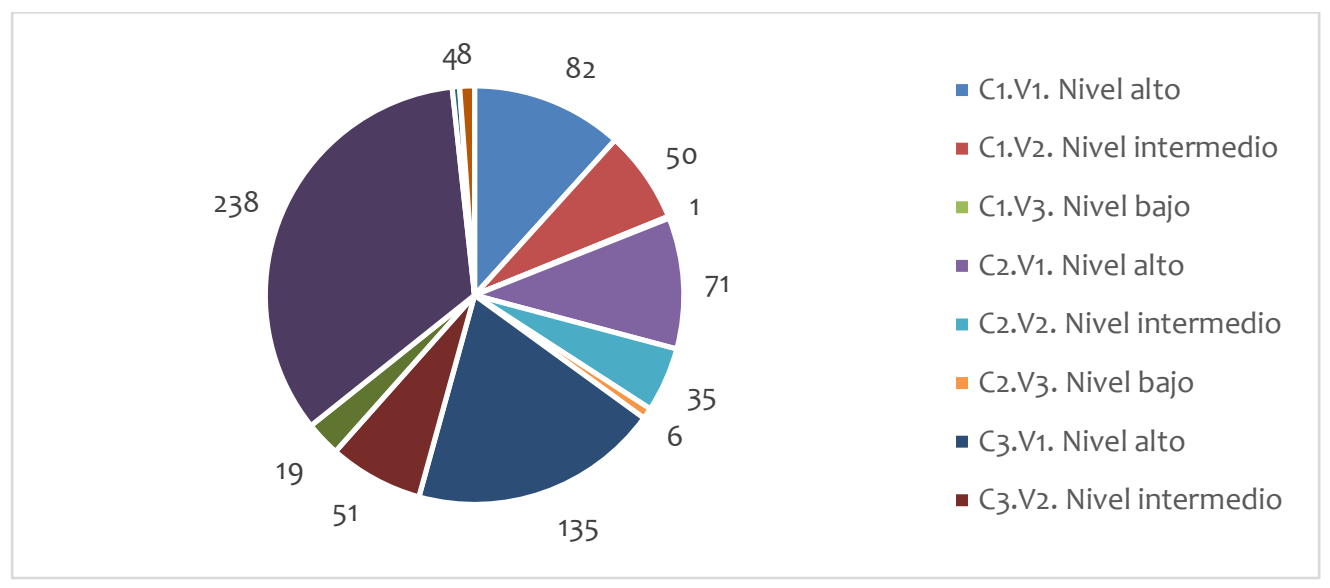

Figura 6. Enraizamiento de códigos (Gr) del cuestionario de preguntas abiertas de EP

En la categoría C1 se registra un nivel alto (C1.V1: Gr=82) e intermedio (C1.V2: Gr=50). El alumnado indica funciones y características de los elementos patrimoniales estudiados. Vinculado al nivel intermedio, reconoce en parte formas de vida de la antigüedad (Neolítico y Edad de Hierro).

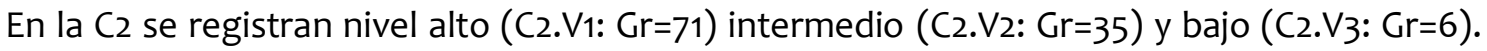
Mencionan diversas acciones negativas que ha sufrido el dolmen: "destruyeron parte del terreno para hacer un muro" (Cita: 8:11). Destaca que varios alumnos/as indican que hay "un cono de violación" (Cita 7:11; 10:11) concepto poco habitual entre el alumnado de esta edad que muestra que han incorporado ciertos conocimientos técnicos.

En la categoría C3 se identifica un considerable nivel alto (C3.V1: $G r=135)$ e intermedio (C3.V2: $\mathrm{Gr}=51$ ). Consideran necesario cuidar el patrimonio, sobre todo por su importancia histórica y por la necesidad de preservarlo: "es importante cuidar el pasado para llevarlo al futuro" (Cita 8:15). Expresan una implicación afectiva y emocional sobre su patrimonio. Mencionan haber hablado del dolmen con su familia, y sienten la necesidad de enseñar cómo hay que protegerlo "todo el mundo debe saber que hay que cuidarlos" (Cita 26:18).

En el Centro 1, respecto a la valoración de la actividad desarrollada sobre música tradicional no hay una respuesta unitaria al identificar si esta música tradicional es conocida en su localidad: cinco creen que sí y cuatro que no. Asumen que si no es conocida es porque "se están perdiendo las tradiciones" (Cita 5:12). Existe casi unanimidad al identificar la música tradicional como algo importante de su pueblo (8/9) ya que "también forma parte del patrimonio" (Cita 5:18). La valoran positivamente no sólo por la dimensión lúdica sino también por su contribución a la conservación y difusión del patrimonio inmaterial: "al escucharlas más gente conocerá estas canciones antiguas"(Cita 6:26) o "porque estamos recuperando el patrimonio"(Cita 5:25).

La satisfacción que muestran sobre los ítems incluidos en la categoría $C_{4}$ es prácticamente total ( $\left.\mathrm{C}_{4} \cdot \mathrm{V} 1: \mathrm{Gr}=238\right)$ o intermedia ( $\left.\mathrm{C}_{4} \cdot \mathrm{V} 2: \mathrm{Gr}=51\right)$ exceptuando alumnos/as a los que no les ha gustado participar en la exposición ( $\left.\mathrm{C}_{3} . \mathrm{V}_{3}: \mathrm{Gr}=19\right)$. Estos aclaran que fue por vergüenza y no por el contenido de la actividad. A todos les gustaría seguir trabajando sobre patrimonio en los siguientes cursos.

Se analizan las reflexiones escritas que responden a la pregunta: ¿Qué es para ti el patrimonio? En la categoría C1 muestran un nivel intermedio de conocimientos sobre el patrimonio próximo (TR.C1.V2: Gr:19). El nivel alto es minoritario (TR.C1.V1: Gr: 2). Expresan un concepto de patrimonio poco preciso: "es toda la cultura de nuestra tierra" (Cita 6:31) o reproducen la definición recibida sin apenas interpretación propia: "son todas las manifestaciones culturales de un lugar, como los monumentos, la música, etc." (Cita 2:33). 
En cuanto a los ejemplos que aportan, el más repetido es el dolmen de la localidad, seguido por la música y las canciones tradicionales. En las reflexiones escritas no se identifica la categoría C3 y sólo en un caso la C2, en un nivel intermedio.

\section{Educación Secundaria}

El alumnado de ES es del Centro 1 (11 alumnos/as). En primer lugar, se resumen los resultados comparativos entre pre-test y post-test de la fase 1.

- Evaluación inicial y formativa

En la categoría C1 disminuye el número de alumnos/as que creen que lo importante de su pueblo es el paisaje que los rodea (del $36,4 \%$ al $16,7 \%$ ) y se incrementan los que señalan los edificios y monumentos visitados (del 9,1\% al 41,7\%). También aumentan quienes consideran que lo más importante para ellos es toda la cultura del pueblo: de $27,3 \%$ al $33,3 \%$; y disminuyen los que no responden (del $27,3 \%$ al $8,3 \%$ ).

En la $C_{2}$ aumentan en un 10\% los que piensan que el cambio más significativo es que desaparecieron muchos edificios antiguos. Descienden en número los que creen que lo que ha cambiado ha sido el edificio del colegio (del $60 \%$ al $41,7 \%$ ). En el post-test, casi la totalidad creen que hay que conservar y conocer los edificios antiguos para saber cómo se vivía. Ninguno de los que proponían darles nuevo uso lo mantiene en el post-test.

Todos suponían que sus abuelos y abuelas condecían importancia a las fotografías únicamente por ser bonitos recuerdos. En el post-test, uno de cada cuatro señala que cuentan historias del pasado del pueblo. Una tercera parte consideraba que era importante sólo como recuerdos personales para las personas que figuran en ellas. En el post-test aumentan los que creen que son importantes para todos porque reflejan nuestro pasado. Mejora la valoración del patrimonio (C3). Aumentan los que lo valoran por qué es importante conservar las obras artísticas como canciones, pinturas, poemas... Aunque la mayoría lo aprecian por ser un legado para las generaciones futuras (58,3\%).

En la categoría C4 el 63, 6\% manifiesta que trabajando con fotografías ha conocido los cambios entre la escuela y los edificios antiguos y actuales. El $27 \%$ va más allá y añaden que estos elementos patrimoniales son importantes para conocer el pasado.

La satisfacción con las actividades es elevada (C5). La mayoría considera interesante identificar los cambios reflejados en las fotografías, y también las canciones de la cantautora $(83,3 \%)$. La realización de vídeos se valora positivamente, no sólo porque haya sido divertida $(16,7 \%)$, sino como herramienta eficaz para difundir su trabajo (83.3\%). Para más de la mitad de los alumnos/as la actividad internivel ha sido satisfactoria. Finalmente, la mitad declara que nadie de su familia había ido a ver su trabajo en la exposición.

- Evaluación sumativa

Fase 2: Los estudiantes cubren, en primer lugar, un cuestionario de preguntas abiertas (Figura 7).

En la categoría C1 el enraizamiento de códigos muestra un nivel intermedio elevado (C1.V2: $\mathrm{Gr}=15)$ seguido del nivel alto ( $\left.\mathrm{C}_{1} . \mathrm{V}_{1}: \mathrm{Gr}=13\right)$ y del nivel bajo $\left(\mathrm{C}_{1}, \mathrm{~V}_{3}: \mathrm{Gr}=2\right)$. Manifiestan que el trabajo con toponimia, música tradicional y cultura castreña les ayudó a aprender: "lo que es el patrimonio y su importancia y la cantidad de monumentos en nuestra localidad" (Cita 9:6). 


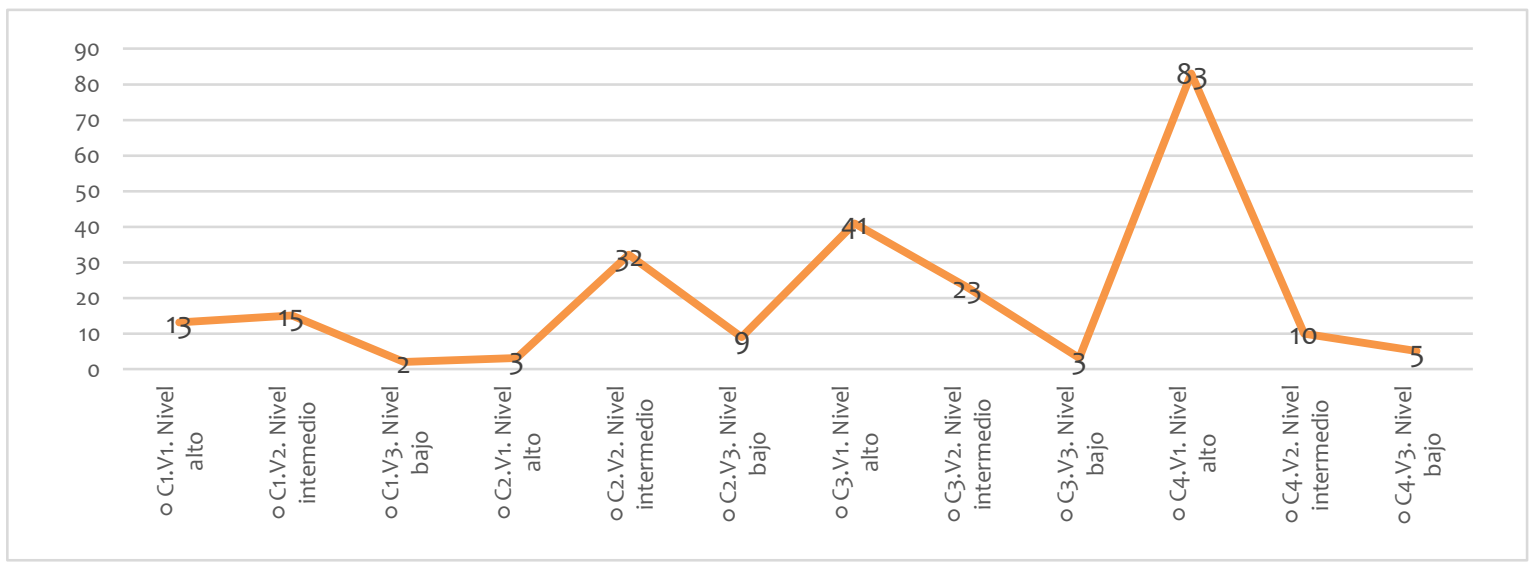

Figura 7. Enraizamiento de códigos (Gr) del cuestionario de preguntas abiertas de ES

En la C2 se encuentra un nivel intermedio: C2.V2: $\mathrm{Gr}=32$. Creen necesario intervenir en la conservación de los castros tanto por la importancia de conservar el patrimonio como por su valor didáctico (10/11).

La categoría $C_{3}$ acoge un nivel considerablemente alto (C3.V1: $\left.\mathrm{Gr}=41\right)$ seguido por el nivel intermedio ( $\left.\mathrm{C}_{3} \mathrm{~V} 2 \mathrm{~V}: \mathrm{Gr}=23\right)$. Consideran que la mayoría de la gente del entorno no conoce el patrimonio local.

La importancia de la difusión vuelve a estar presente en la C4. Consideran útiles las actividades de transmisión de conocimientos. La valoración de la metodología es mayoritariamente alta ( $\mathrm{C} 4 . \mathrm{V} 1: \mathrm{Gr}=83)$. Existe división de opiniones al tener que decidir si les gustaría trabajar en los siguientes cursos sobre patrimonio. Los que quieren continuar (5) valoran positivamente lo mucho que aprenden, además de relacionarlo con dar continuidad a las tradiciones. Los otros cinco que contestan manifiestan que les quitan tiempo para otras materias: "perdemos muchas clases y en estas tuvimos que darnos prisa para hacer los trabajos" (Cita 3:31; 11:31; 8:32).

En el último instrumento para ES se pide que graben vídeo-cápsulas con su definición de patrimonio (6/11). Se observan definiciones del patrimonio formales que no permiten apreciar enraizamiento de códigos en la categoría C1. Cuatro coinciden en afirmar que son todas las cosas que se conservan y sirven para conocer el pasado. Cuatro ( 2 2.V2. Gr=4) destacan su importancia para saber de dónde venimos para poder comprender lo que somos y por qué (nivel intermedio). La categoría C3 se sitúa entre un nivel intermedio ( $C_{3} . V_{2}$ : $\left.G r=4\right)$ y bajo (C3.V3: Gr=2).

\section{Discusión y conclusiones}

Este estudio se ha propuesto como objetivos conocer los resultados de aprendizaje del alumnado participante en el programa Patrimonializarte y su valoración de la metodología utilizada para analizar su eficacia, eficiencia y efectividad, así como su impacto y el nivel de satisfacción de los implicados.

El impacto del programa ha sido mayor en El. Algunos de los alumnos/as ya habían visitado ciertos elementos patrimoniales, como el dolmen, en campamentos de verano, pero manifiestan que no les habían explicado nada sobre él. La educación patrimonial no sólo pasa por visitar vestigios, sino que necesita proyectos integrales. La concienciación es primordial para despertar sentido crítico con la conservación y preservación del patrimonio. Así lo transmiten los alumnos, que han interiorizado la importancia de cuidar y conservar su 
patrimonio sintiendo la necesidad de difundir todo lo que han aprendido entre su familia y la localidad (Monti et al., 2017). Las emociones son clave para esta interiorización. El alumnado desarrolla un sentimiento de identidad con su patrimonio al conocer las malas condiciones de conservación (Peinado, 2020). Así se revela en los tests proyectivos gráficos: la mayoría dibujan el dolmen padeciendo agresiones. Los niños adquieren un sentimiento de apego y muestran una predisposición a conocer más el patrimonio; todos quieren seguir trabajando este tema en cursos posteriores. La capacidad de aprendizaje del paso del tiempo y de la historia es alta en estas edades: incorporaban nuevos conocimientos sobre el patrimonio próximo desde la primera sesión y se esfuerzan en mostrar su concienciación con su conservación que muestran también en sus dibujos, incluyendo en ellos las piedras rotas o el corredor desaparecido (Miralles y Rivero, 2012).

En EP, se refuerzan conocimientos sobre el patrimonio, especialmente con el próximo, muy desconocido para ellos, como muestran los resultados. Esto contrasta con resultados de estudios sobre futuros profesores (Gómez-Carrasco, Miralles-Martínez, Fontal, e IbáñezEtxeberria, 2020). Tras la implementación del programa, el alumnado se identifica con lo propio, se preocupa por el patrimonio inmaterial y aboga por su preservación para futuras generaciones. Parece recomendable, pues, trabajar en esta línea (Kirshenblatt-Gimblett, 2004; Lenzerini, 2011).

En ES la suma de conocimientos complementarios (toponimia, música, espacio geográfico, historia, integración de las TIC) ha generado un conocimiento integral que contribuye a la valoración de elementos patrimoniales próximos. Atribuyen gran importancia a la difusión de información con herramientas y métodos variados para compartir con la población local la responsabilidad de cuidar el patrimonio. Discrepan entre ellos sobre si seguir con la educación patrimonial en cursos posteriores porque consideran que este tipo de programas restan tiempo para otras materias. Esta opinión corresponde a una perspectiva academicista tradicional: lo que no está en el currículo oficial es una relativa pérdida de tiempo. Por eso consideramos necesaria la inclusión de la educación patrimonial de manera normalizada en el currículum, para que el alumnado comprenda su relevancia e incremente su interés (Cuenca-López, Estepa-Giménez, y Martín-Cáceres, 2017; Cuenca-López y López-Cruz, 2014; Estepa et al., 2011).

Se ha constatado la eficacia del programa Patrimonializarte: se han logrado los objetivos previstos en el programa; su eficiencia: los objetivos se han alcanzado con éxito con los recursos y medios previstos; y efectividad: se han logrado efectos beneficiosos no previstos en el programa, como el impacto en agentes externos al programa (familias) o la adquisición de una conciencia de conservación tan arraigada en El. El nivel de satisfacción del alumnado con las actividades desarrolladas es alto. Se han adquirido nuevos conocimientos sobre el patrimonio próximo desde la educación infantil a la educación secundaria y se ha obtenido un nivel alto de concienciación con el patrimonio.

\section{Referencias}

Calaf, R., Gutiérrez, S., y Suárez, M.A. (2020). La evaluación en la Educación Patrimonial. 20 años de investigaciones y Congresos de ICOM. Aula Abierta, 49(1), 55-64. doi: 10.17811/rifie.49.1.2020.55-64

Castro-Calviño, L., y López-Facal, R. (2019). Educación patrimonial: necesidades sentidas por el profesorado de infantil, primaria y secundaria. RIFOP, 33(1), 97-114. Recuperado de https://recyt.fecyt.es/index.php/RIFOP/article/view/72020 
Castro-Calviño, L., Rodríguez-Medina, J., Gómez-Carrasco, C. J., y López-Facal, R. (2020). Patrimonializarte: A Heritage Education Program Based on New Technologies and Local Heritage. Education Sciences, 10(7), 176. doi: 10.3390/educsci10070176

Cook, T. D., y Reichardt, CH. S. (1982). Qualitative and Quantitative Methods in Evaluation Research. Londres, Reino Unido: Sage Publications.

Cuenca-López, J. M. (2003). Análisis de concepciones sobre la enseñanza del patrimonio en la educación obligatoria. Enseñanza de las Ciencias Sociales: Revista de Investigación, (2), 37-45. Recuperado de https://www.raco.cat/index.php/EnsenanzaCS/article/view/126155

Cuenca-López, J. M., y López-Cruz, I. (2014). Teaching heritage in Social Science, Geography and History textbooks in Compulsory Secondary Education. Culture and Education, 26(1), 1-43. doi: 10.1080/11356405.2014.908663

Cuenca-López, J. M., Estepa-Giménez, J., y Martín-Cáceres, M. J. (2017). Patrimonio, educación, identidad y ciudadanía. Profesorado y libros de texto en la enseñanza obligatoria. Revista de Educación, (375), 136-159. doi: 10.4438/1988-592X-RE-2016-375338

Cuenca-López, J. M., Martín Cáceres, M., y Estepa, J. (2020). Buenas prácticas en educación patrimonial. Análisis de las conexiones entre emociones, territorio y ciudadanía. Aula Abierta, 49(1), 45-54. doi: https://doi.org/10.17811/rifie.49.1.2020.45-54

Delgado-Algarra, E. J., y Cuenca-López, J. M. (2020). Challenges for the Construction of Identities With Historical Consciousness: Heritage Education and Citizenship Education. En E. J. Delgado-Algarra y J. M. Cuenca-López. (Eds.), Handbook of Research on Citizenship and Heritage Education (pp.1-15). Hershey, EE. UU: IGI Global.

Domínguez, A., y López-Facal, R. (2015). Patrimonio, entorno y procesos de identificación en la educación primaria. CLIO. History and History teaching, (41). Recuperado de http://clio.rediris.es/n41/articulos/mono/MonFacal2015.pdf

Escudero, T. (2016). La investigación evaluativa en el siglo XXI: un instrumento para el desarrollo educativo y social cada vez más relevante. RELIEVE, 22(1), 1-20. doi: 10.7203/relieve.22.1.8164

Estepa, J., Ferreras, M., López, I., y Morón, H. (2011). Análisis del patrimonio presente en los libros de texto: obstáculos, dificultades y propuestas. Revista de Educación 2011, (355), 573-589. doi: 10-4438/1988-592X-RE-2011-355-037

Fetterman, D. M., Kaftarian, S. J., y Wandersman, A. (2015). Empowerment Evaluation: Knowledge and Tools for Self-Assessment, Evaluation Capacity Building, and Accountability. Thousand Oaks, California, EE. UU: Sage Publications. doi: $10.4135 / 9781483387079$

Fontal, O. (2003). La educación patrimonial: Teoría y práctica para el aula, el museo e Internet. Gijón, España: Trea.

Fontal, O. (2016). Educación patrimonial: retrospectiva y prospectivas para la próxima década. Estudios Pedagógicos, 42(2), 415-136. doi: 10.4067/S071807052016000200024 .

Fontal, O., Ibáñez-Etxeberria, A., Martínez, M., y Rivero, P. (2017). El patrimonio como contenido en la etapa de Primaria: del curriculum a la formación de maestros. REIFOP, 20(2), 79-95. doi: 10.6018/reifop/20.2.286321 
Fontal, O., y Martínez, M. (2017). Evaluación de programas educativos sobre Patrimonio Cultural Inmaterial. Estudios pedagógicos, 43(4), 69-89. doi: 10.4067/S071807052017000400004

Fontal, O., y García, S. (2019). Evaluación de programas de Educación Patrimonial: estándares de calidad. ENSAYOS, Revista de la Universidad de Albacete, 34(1), 1-15. doi: 10.18239/ENSAYOS.V34l1.2039

Fontal, O., García, S., Arias, B., y Arias, V. (2019). Evaluación de la calidad de programas de educación patrimonial: construcción y calibración de la escala Q-Edutage. Revista de Psicodidáctica, 24(1), 31-38. doi: 10.1016/j.psicod.2018.07.003

Gómez-Carrasco, C. J., Miralles-Martínez, P., Fontal, O., e Ibáñez-Etxeberria, A. (2020). Cultural Heritage and Methodological Approaches-An Analysis through Initial Training of History Teachers (Spain-England). Sustainability, 12(3), 933. doi: $10.3390 /$ su12030933

Greene, J. C. (2005). The generative potential of mixed methods inquiry. International Journal of Research \& Method in Education, 28(2), 207-211. doi: 10.1080/01406720500256293

ICOMOS. (2017). Delhi Declaration on Heritage and Democracy. Recuperado de https://www.icomos.org/images/DOCUMENTS/Charters/GA2017_DelhiDeclaration_20180117_EN.pdf

Jiménez-Pérez, R.; Cuenca-López, J. M., y Ferreras-Listán, M. (2010). Heritage education: Exploring the conceptions of teachers and administrators from the perspective of experimental and social science teaching. Teaching and Teacher Education, 26(6), 13191331. doi: 10.1016/j.tate.2010.01.005

Johnson, R. B., y Onwuegbuzie, A. J. (2004). Mixed Methods Research: A Research Paradigm Whose Time Has Come. Educational Researcher, 33(7), 14-26. Recuperado de http://sites.uci.edu/socscihonors/files/2017/09/Mixed_Methods_Research.pdf

Kirshenblatt-Gimblett, B. (2004). Intangible Heritage as Metacultural Production. Museum International, 56(1-2), 52-56. doi: 10.1111/j.1350-0775.2004.00458.x

Lenzerini, F. (2011). Intangible Cultural Heritage: The Living Culture of Peoples. European Journal of International Law, 22(1), 101-120. doi: 10.1093/ejil/chroo6

Marqués, X., Castro-Fernández, B., y López-Facal, R. (2020). Patrimonio y comunidad patrimonial: construcción de una identidad compartida en un entorno rural. Aula Abierta, 49(1), 25-34. doi: 10.17811/rifie.49.1.2020.25-34

Martín-Cáceres, M. J., y Cuenca-López, J. M. (2015). Educomunicación del patrimonio. Educatio Siglo XXI, 33(1), 33-54. doi: 10.6018/j/222491

Miralles, P., y Rivero, P. (2012). Propuestas de innovación para la enseñanza de la historia en Educación Infantil. REIFOP, 15(1), 81-90. Recuperado de https://www.redalyc.org/articulo.oa?id=217024398006

Monti, F., Farné, R., Crudeli, F., Agostini, F., Minelli, M., y Ceciliani, A. (2017). The role of Outdoor Education in child development in Italian nursery school. Early Child Development and Care, 189(6), 867-882. doi: 10.1080/03004430.2017.1345896

Peinado, M. (2020). Del patrimonio a la ciudadanía en Educación Infantil. Investigación en la Escuela, (101), 48-57. doi: 10.12795/IE.2020.1101.04

Pérez, R. (2002). La evaluación de programas en el marco de la educación de calidad. En-clave pedagógica,

43-76. Recuperado

de http://www.uhu.es/publicaciones/ojs/index.php/xxi/article/view/620 
Pérez, R. (2006). Evaluación de programas educativos. Madrid, España: Muralla.

Pinto, H., y Zarbato, J. (2017). Construyendo un aprendizaje significativo a través del patrimonio local: prácticas de Educación patrimonial en Portugal y Brasil. Estudios Pedagógicos, 43(4), 203-227. doi: 10.4067/S0718-07052017000400011

Sánchez-Macías, I., Fontal, O., y Rodríguez-Medina, J. (2019). La evaluación de Aprendizajes: una práctica pendiente en Educación Patrimonial. RIFOP, 33(2), 163-186. doi: 10.47553/rifop.v33i2.73325

Sánchez, M. F., y Murga-Menoyo, M. A. (2019). Place-Based Education: una estrategia para la sostenibilización curricular de la educación superior. Bordón. Revista de pedagogía, $71(2)$ 155-174.

Recuperado

de https://recyt.fecyt.es/index.php/BORDON/article/view/73021

Stake, R. E. (1995). The art of case study research. Londres, Reino Unido: Sage Publications.

Trabajo-Rite, M., y Cuenca-López, J. M. (2020). Student concepts after a didactic experiment in heritage education. Sustainability, 12(7), 3046. doi: 10.3390/su12073046

UNESCO. (2003). Convención para la Salvaguardia del Patrimonio Cultural Inmaterial. Recuperado de https://unesdoc.unesco.org/ark:/48223/pfo000132540_spa

Woodhouse, J. L., y Knapp, C. E. (2000). Place-based Curriculum and Instruction: Outdoor and Environmental Education Approaches. Champaign, EE. UU: ERIC Digest.

Yang, Y., Shafi, M., Song, X., y Yang, R. (2018). Preservation of cultural heritage embodied in traditional crafts in the developing countries. A case study of Pakistani handicraft industry. Sustainability, 10(5), 1336. doi: 10.3390/su10051336 